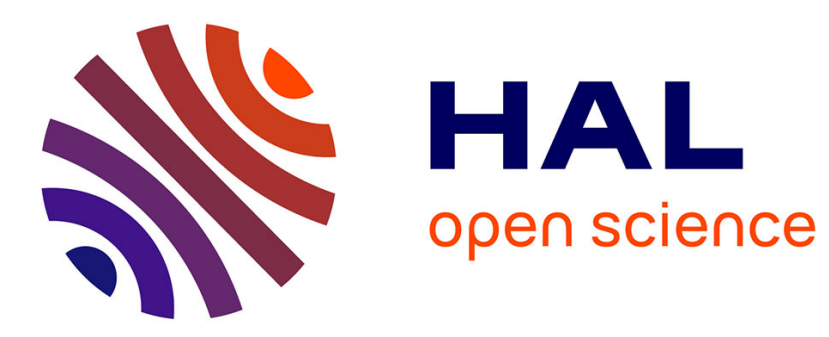

\title{
Robust control for an off-centered quadrotor
}

Thibaut Raharijaoana, François Bateman

\section{To cite this version:}

Thibaut Raharijaoana, François Bateman. Robust control for an off-centered quadrotor. 19th Mediterranean Conference on Control \& Automation (MED), Jun 2011, Corfu, Greece. 10.1109/MED.2011.5983171 . hal-00830136

\section{HAL Id: hal-00830136 https://inria.hal.science/hal-00830136}

Submitted on 4 Jun 2013

HAL is a multi-disciplinary open access archive for the deposit and dissemination of scientific research documents, whether they are published or not. The documents may come from teaching and research institutions in France or abroad, or from public or private research centers.
L'archive ouverte pluridisciplinaire HAL, est destinée au dépôt et à la diffusion de documents scientifiques de niveau recherche, publiés ou non, émanant des établissements d'enseignement et de recherche français ou étrangers, des laboratoires publics ou privés. 


\title{
Robust control for an off-centered quadrotor
}

\author{
Thibaut Raharijaona and François Bateman
}

\begin{abstract}
The development of quadrotor unmanned aerial vehicles -UAVs- in potential civil applications is conditioned by the embedded elements such as removable payload, miniature actuators, sensors and power storage. As simple as the structure of the quarotor is, the dynamic behavior is complex.

The paper details a model of a quadrotor which considers the effects of the location of the center of gravity and the gyroscopic torques due to the derivative of the speed propellers. A simple control law in order to regulate the angular velocities and to track angular references is designed. Time domain constraints and parameters which belong to a polytope define the basis of the regulator synthesis. The relevance of the study is fulfilled thanks to numerical simulations and experimental plateform is presented.
\end{abstract}

\section{INTRODUCTION}

Underactuated quadrotor UAV is over the last years the subject to the developments of numerous challenging studies. Due to the progress in sensing technologies, power storage and miniature actuators, the cost and size reductions for miniUAV lead professionals and researchers to several applications [1], [2], [3], [4].

Research activities focused on modelling and control design of this aerial platform [5], [6]. More precisely, the dynamic description of the quadrotor in mechanical and aerodynamic senses is still a sensitive subject. As briefly depicted in [7], the location of the center of gravity c.g. given by the battery, sensors, on-board computer and eventually detachable payload such as camera, affects the stability and the dynamic performances of the quadrotor.

In this paper, the idea of developing an off-centered c.g. model of the aerial vehicle and describing the gyroscpic torques due to the time derivative of propeller speeds arises from an industrial application which aims at supervising possible damage into civil buildings using removable cameras and leads to take into account unexpected dynamic behavior for the control.

Concerning the control, many solutions are proposed in the literature and are illustrated with respect to a dedicated small platform quadrotor UAV [8], [9]. For known or uncertain parameters such as various masses on board which modify the location of c.g., the design of a simple control inspired by stability guarantee and dynamic constraints is proposed. The paper is organized as follows.

An off-centered c.g. dynamic model of the UAV with the gyroscopic torques due to the time derivative of the speed propellers is detailed in Section II. We obtain a six DOF

T. Raharijaona is with the LSIS laboratory, Aix-Marseille University, FRANCE thibaut.raharijaonalsis.org

F. Bateman is with the LSIS laboratory, Aix-Marseille University, FRANCE francois.batemanelsis.org model with four inputs being the rotation speeds of the propellers and we consider six outputs which consist in the angular velocities and the angular positions. In Section III, a state-feedback based control with PI controller which takes into account the domain of a set of varying parameters is investigated. In Section IV, we expose numerical simulations and results. We discuss the relevance of the study. Section $\mathrm{V}$ is dedicated to the presentation of our experimental benchmark which is the basis of our further developments.

\section{DYNAMIC MODEL OF THE UAV}

Quadrotor theory has widely been studied in the literature. However some phenomenons such as an off-centered center of gravity c.g. or the gyroscopic torques due to the time derivative of propeller speeds are not tackled. In particular, the use of detachable payloads may affect the position of c.g. and the moments of inertias. A model of a quadrotor taking into account these various effects is proposed.

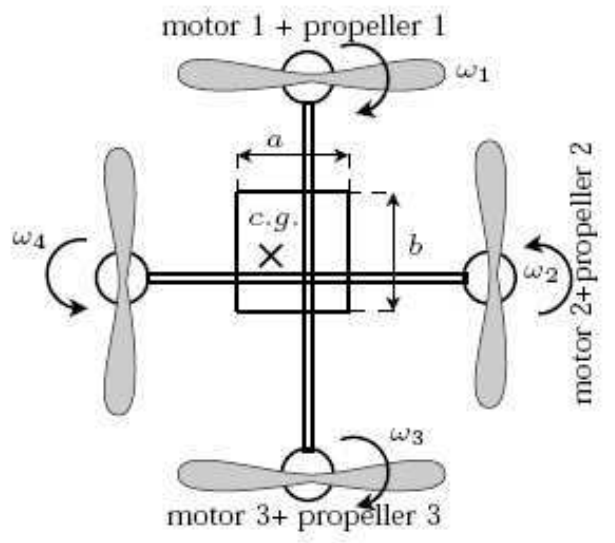

Fig. 1. Top view of the quadrotor

The model of the quadrotor is described with a six degrees of freedom platform. The following dynamic model of the quadrotor is presented in the case of a rigid-body, the weight $m$ is constant and the center of gravity c.g. is a fixed position but it depends on the payload position. Let $R_{E}=\left(O, \overrightarrow{x_{E}}, \overrightarrow{y_{E}}, \overrightarrow{z_{E}}\right)$ be a right-hand inertial frame such that $\overrightarrow{z_{E}}$ is the vertical direction downwards the earth, $\vec{\xi} R_{E}=(x, y, z)^{T}$ denotes the position of $c . g$. in $R_{E}$. Let $R_{b}=\left(c . g ., \overrightarrow{x_{b}}, \overrightarrow{y_{b}}, \overrightarrow{z_{b}}\right)$ be a right-hand body fixed frame for the UAV, at $t=0 R_{E}$ and $R_{b}$ coincide. The linear velocities $\vec{\mu}^{R_{b}}=(u, v, w)^{T}$ and the angular velocities $\vec{\Omega}^{R_{b}}=(p, q, r)^{T}$ are expressed in the body frame $R_{b}$ where $p, q, r$ are roll, pitch and yaw angular velocities, respectively. The orientation of the rigid body in $R_{E}$ is located with the bank angle $\varphi$, the pitch angle $\theta$ and the heading angle $\psi$. The 
transformation from $R_{b} \rightarrow R_{E}$ is given by a transformation matrix $T_{b E}$ given in the appendix. With respect to the body frame, the propeller speeds $\omega_{1}, \omega_{3}<0$ and $\omega_{2}, \omega_{4}>0$

\section{A. Mass and moment of inertia}

Let $m_{m}, m_{c}, m_{p}$ the weight of a motor, the mass of the cross and the mass of the payload respectively, then $m=$ $4 m_{m}+m_{c}+m_{p}$. The coordinates of $c . g$. of the payload are $(\alpha, \beta, \gamma)^{T}$ and the coordinates of $c . g$. of the quadrotor $\left(x_{c g}, y_{c g}, z_{c g}\right)^{T}$ are detailed in the appendix.

Huygens's theorem is used to calculate the inertia matrix $J$ with respect to the quadrotor c.g.. For an off-centered c.g., the inertia matrix writes:

$$
\left(\begin{array}{ccc}
J_{x} & -J_{x y} & -J_{x z} \\
-J_{x y} & J_{y} & -J_{y z} \\
-J_{x z} & J_{x y} & J_{z}
\end{array}\right)
$$

The inertia moments and inertia cross-product are detailed in the appendix.

\section{B. Translational and rotational motion equations}

According to Newton's second law:

$$
\begin{gathered}
\vec{F}^{R_{b}}=m\left(\frac{d \vec{\mu}^{R_{b}}}{d t}+\vec{\Omega}_{R_{b} / R_{E}}^{R_{b}} \times \vec{\mu}^{R_{b}}\right) \\
\dot{u}=\frac{F_{x}^{R_{b}}}{m}-q w+r v \\
\dot{v}=\frac{F_{y}^{R_{b}}}{m}+p w-r u \\
\dot{w}=\frac{F_{z}^{R_{b}}}{m}-p v+q u
\end{gathered}
$$

Forces $F_{x}, F_{y}, F_{z}$ acting on the quadrotor are expressed in $R_{b}$, they are due to gravity $F_{\text {grav }}$, to propulsion $F_{\text {prop }}$, and to aerodynamic effects $F_{\text {aero }}$.

The relationships between the angular velocities, their derivatives and the moments $\vec{M}^{R_{b}}=\left(\mathcal{M}_{x}, \mathcal{M}_{y}, \mathcal{M}_{z}\right)^{T}$ applied to the aircraft originate from the general moment equation. Let $\vec{\sigma} R_{b}$ the kinetic momentum, $J$ the inertia matrix, $I_{p}$ the propeller inertia and $\times$ the cross product.

$$
\vec{\sigma}^{R_{b}}=J \vec{\Omega}^{R_{b}}+I_{p} \sum_{i=1}^{4}(-1)^{i} \omega_{i} \overrightarrow{z_{b}}
$$

the general moment equation:

$$
\begin{gathered}
\dot{\vec{\sigma}}^{R_{b}}=\vec{M}^{R_{b}}-\vec{\Omega}^{R_{b}} \times \vec{\sigma}^{R_{b}} \\
\left(\begin{array}{c}
\dot{p} \\
\dot{q} \\
\dot{r}
\end{array}\right)=J^{-1}\left[\left(\begin{array}{l}
\mathcal{M}_{x} \\
\mathcal{M}_{y} \\
\mathcal{M}_{z}
\end{array}\right)-\left(\begin{array}{c}
p \\
q \\
r
\end{array}\right) \times\left[J\left(\begin{array}{c}
p \\
q \\
r
\end{array}\right)\right.\right. \\
\left.\left.-I_{p}\left(\begin{array}{c}
0 \\
0 \\
\sum_{i=1}^{4}(-1)^{i} \dot{\omega}_{i}
\end{array}\right)+I_{p}\left(\begin{array}{c}
0 \\
\sum_{i=1}^{4}(-1)^{i} \omega_{i}
\end{array}\right)\right]\right]
\end{gathered}
$$

The moments $\left(\mathcal{M}_{x}, \mathcal{M}_{y}, \mathcal{M}_{z}\right)$ proceed from the differential of forces due to the difference between propeller speeds. It also exists gyroscopic torques due to angular velocity crosscouplings and to angular velocity time derivatives.
1) Model of the forces:

$$
\begin{aligned}
& F_{\text {grav }}^{R_{E}}=\left(\begin{array}{lll}
0 & 0 & g
\end{array}\right)^{T} \\
& F_{\text {prop }}^{R_{b}}=\left(\begin{array}{lll}
0 & 0 & -\sum_{i=1}^{4} k \omega_{i}^{2}
\end{array}\right)^{T}
\end{aligned}
$$

$g$ is the acceleration of free fall and $k$ is a constant which depends on the propeller shape and the air density [7].

2) Model of the torques: The off-centered center of gravity affects the roll and pitch torques due to the difference between propeller speeds:

$$
\begin{aligned}
& \mathcal{M}_{x}=k \omega_{1}^{2} y_{c g}-k \omega_{2}^{2}\left(l-y_{c g}\right)+k \omega_{3}^{2} y_{c g}+k \omega_{4}^{2}\left(l+y_{c g}\right) \\
& \mathcal{M}_{y}=k \omega_{1}^{2}\left(l-x_{c g}\right)-k \omega_{2}^{2} x_{c g}-k \omega_{3}^{2}(l+x)-k \omega_{4}^{2} x_{c g}
\end{aligned}
$$

As far as the yaw torque $\mathcal{M}_{z}$ is concerned, it proceeds

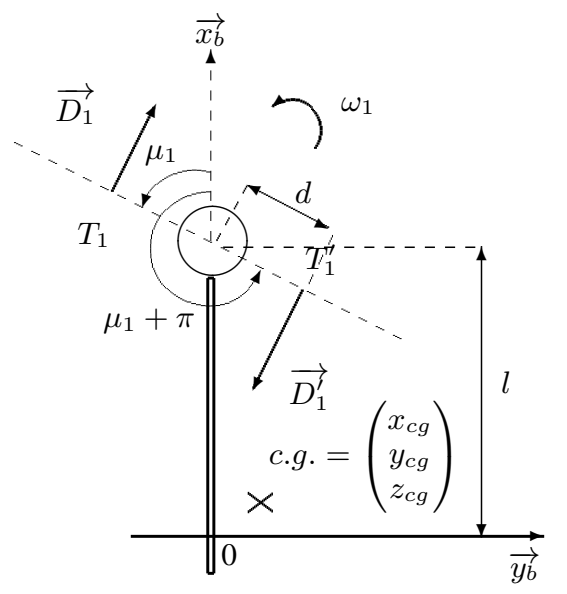

Fig. 2. Top view of the first propeller

from Fig. 2. For the propeller 1 , as shown in Fig. 2, $D_{1}$ and $D_{1}^{\prime}$ are the drag forces and $T_{1}, T_{1}^{\prime}$ are their application points with $T_{1} T_{1}^{\prime}=2 d$. The drag force is proportional to the square of the propeller speed [7], then the blades being symmetrical, assume that $D_{1}=D_{1}^{\prime}=\delta \omega_{1}^{2}$. At $t=0$ the propeller blade angular positions are $\mu_{1}$ and $\mu_{1}+\pi$. Next $\overrightarrow{T_{1} c g}, \overrightarrow{T_{1}^{\prime} c g}$ coordinates are:

$$
\begin{aligned}
& \overrightarrow{T_{1} c g}=\left(\begin{array}{c}
l+d \cos \left(\omega_{1} t+\mu_{1}\right)-x_{c g} \\
-d \sin \left(\omega_{1} t+\mu_{1}\right)-y_{c g} \\
-z_{c g}
\end{array}\right) \\
& \overrightarrow{T_{1}^{\prime} c g}=\left(\begin{array}{c}
l-d \cos \left(\omega_{1} t+\mu_{1}\right)-x_{c g} \\
d \sin \left(\omega_{1} t+\mu_{1}\right)-y_{c g} \\
-z_{c g}
\end{array}\right)
\end{aligned}
$$

For each blade, the drag projected on axis $\overrightarrow{x_{b}}$ and $\overrightarrow{y_{b}}$ writes:

$$
\overrightarrow{D_{1}}=\left(\begin{array}{c}
D_{1} \sin \left(\omega_{1} t+\mu_{1}\right) \\
D_{1} \cos \left(\omega_{1} t+\mu_{1}\right) \\
0
\end{array}\right) \quad \overrightarrow{D_{1}^{\prime}}=\left(\begin{array}{c}
-D_{1} \sin \left(\omega_{1} t+\mu_{1}\right) \\
-D_{1} \cos \left(\omega_{1} t+\mu_{1}\right) \\
0
\end{array}\right)
$$

For this propeller, the resulting moment is a yaw moment:

$$
\overrightarrow{T_{1} c g} \times \overrightarrow{D_{1}}+\overrightarrow{T_{1}^{\prime} c g} \times \overrightarrow{D_{1}^{\prime}}=\left(\begin{array}{c}
0 \\
0 \\
2 d D 1
\end{array}\right)
$$


By reaction, the yaw torque produced on the platform is equal to $-2 d D_{1}=-2 d \delta \omega_{1}^{2}$. As propeller 3 rotates in the same direction and propellers 2 and 4 rotate in opposite direction, the yaw torque is:

$$
\mathcal{M}_{z}=-2 d \delta \omega_{1}^{2}-2 d \delta \omega_{3}^{2}+2 d \delta \omega_{2}^{2}+2 d \delta \omega_{4}^{2}
$$

It appears that the yaw torque is independent of the center of gravity position. However, due to the sticks of the cross, the airflow is disturbed when a blade crosses over it and the calculation reveals an oscillatory torque which produces mechanical vibrations.

3) Kinematic relations: It is also necessary to be able to track the flight path relative to earth. The kinematic relations are given by:

$$
\begin{aligned}
\dot{T}_{b E} & =T_{b E} s k(\vec{\Omega}) \\
\dot{\vec{\xi}} & =T_{b E} \vec{v}
\end{aligned}
$$

$\operatorname{sk}(\vec{\Omega})$ is the skew-symmetric matrix such that $\operatorname{sk}(\vec{\Omega}) \vec{\epsilon}=$ $\vec{\Omega} \times \vec{\epsilon}$ for any $\epsilon \in \mathbb{R}^{3}$. However, the quadrotor stabilization problem is first an attitude control problem, thus the linear velocities $\vec{\mu}$ and cartesian coordinates $\vec{\xi}$ are not studied in the sequel. Assume that the chosen state vector $x=$ $[\phi, \theta, \psi, p, q, r]^{T}$ is measured. From (6), the nonlinear model of the quadrotor can be written as:

$$
\left\{\begin{array}{l}
\dot{x}=f(x, u) \\
y=x
\end{array}\right.
$$

with $u(t) \in \mathbb{R}^{4}$ is the control input vector and stands for the rotation speed of the four propellers. $\vec{\Omega}^{R_{E}}=[\dot{\phi}, \dot{\theta}, \dot{\psi}]^{T}=$ $R_{r} \vec{\Omega} R_{b}$ where $R_{r}$ is given by:

$$
\left(\begin{array}{ccc}
1 & \sin (\varphi) \tan (\theta) & \cos (\varphi) \tan (\theta) \\
0 & \cos (\varphi) & -\sin (\varphi) \\
0 & \frac{\sin (\varphi)}{\cos (\theta)} & \frac{\cos (\varphi)}{\cos (\theta)}
\end{array}\right)
$$

\section{ROBUST CONTROL IN LMI REGION}

The purpose of the control design proposed in this paper, is first to perform the angular velocity regulation and second to track an angular predefined trajectory given by the path $\left[\phi_{\text {ref }}, \theta_{\text {ref }}, \psi_{\text {ref }}\right]^{T}$ with the smallest tracking error. Robust stability and performances are ensured for a convex set of parameters thanks to a robust pole placement in LMI region.

From the linear approximation around an equilibrium point of the UAV, one can define the following state space representation which stands for a Linear Parameter Varying (LPV) system:

$$
\left\{\begin{array}{l}
\dot{x}=A(\rho) x(t)+B_{u}(\rho) u(t)+B_{e}(\rho) e(t) \\
y(t)=C_{y}(\rho) x(t) \\
z(t)=C_{z}(\rho) x(t)
\end{array}\right.
$$

The state vector is $x \in \mathbb{R}^{6 \times 1}$ such that $x=$ $[\phi, \theta, \psi, p, q, r]^{T} . \phi, \theta, \psi$ are the angles around the axes as previously defined and $p, q, r$ are the angular velocities in the application. $A(\rho) \in \mathbb{R}^{6 \times 6}, B_{u}(\rho)=B_{e}(\rho)=\left[b_{1}, b_{2}, b_{3}, b_{4}\right]$ where $b_{i} \in \mathbb{R}^{6 \times 1}$ and $C_{y}(\rho)=C_{z}(\rho)=I_{6 \times 6} \cdot y(t)$ is the measured output vector and $z(t)$ is the controlled output vector which corresponds to the state $x=[\phi, \theta, \psi, p, q, r]^{T}$. $e(t)$ is the exogenous input which stands for disturbances. $\rho$ is the vector of parameters which is detailed in the following. $\left(A(\rho), B_{u}(\rho)\right)$ corresponds to a set of equimibrium points which corresponds to the attitude of the UAV. The schematic diagram of the autonomous flight control for the generalized plant is presented in Fig. 3.

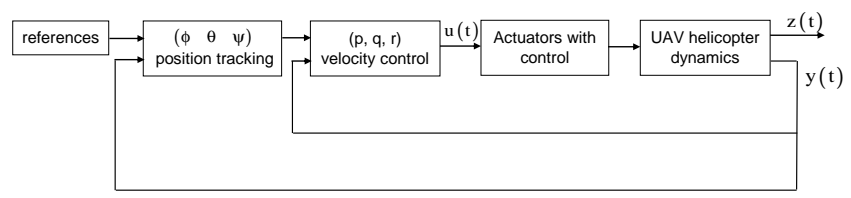

Fig. 3. Generalized plant with control

\section{A. Parametrization of the polytopic system}

In practice, the helicopter is designed to perform flight missions with various carrying capacity on board. The mentioned load consists in a set of cameras which are fixed on the frame of the helicopter. Therefore, the position and the mass of the onboard load define a set of parameters. More precisely, the load position along longitudinal axis is only considered. Consequently, the vector of parameters $\rho$ is defined such that:

$$
\rho=\left(x_{C G}, m_{p}\right)
$$

where $x_{C G}$ is the position of the load along the longitudinal axis and $m_{p}$ is its mass.

In order to build the vertices of the polytopic system for the UAV, we represent in Fig. 4 thanks to the points whose the coordinates are given by $\left(x_{C G}, m_{p}\right)$, the feasible configurations. The points are interpolated by solid lines.

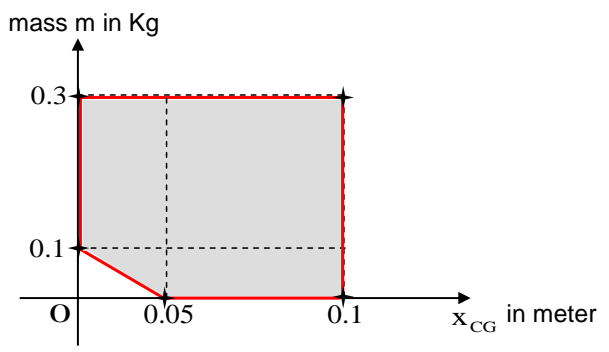

Fig. 4. Vertices of the polytopic system for the parameters $x_{C G}$ and $m_{p}$

From the convex polytope drawn in solid lines in Fig. 4, we define in Table I, the vertices of the polytopic system. One can remark that each vertex corresponds to a feasible configuration of the quadrotor in the position of the center of gravity $x_{C G}$ and the mass $m_{p}$.

TABLE I

COORDINATES OF THE VERTICES OF THE POLYTOPIC SYSTEM

\begin{tabular}{|l||l|l|l|l|l|}
\hline$x_{C G}$ in meter & 0.0 & 0.0 & 0.1 & 0.1 & 0.05 \\
\hline$m_{p}$ in $\mathrm{Kg}$ & 0.1 & 0.3 & 0.3 & 0.0 & 0.0 \\
\hline
\end{tabular}




\section{B. Control objectives}

The control design proposed in this paper aims at ensuring asymptotic stability of the UAV quadrotor for a convex set of parameters which describes a polytope. The angular velocities are regulated and the angular positions track flight references. The variables to be controlled are the roll attitude $(\phi)$, the pitch $(\theta)$, the yaw $(\psi)$ and the angular velocities $p$, $q, r$.

The control objectives are formulated such that:

- The closed-loop system is defined thanks to (17) and the state feedback control input $u=K x$ is locally stable. $K$ is a constant matrix gain and the controller is not gain-scheduled.

- The closed-loop tracking of angular references expects no errors.

- The $H_{2}$ norm of the transfer functions (from (17)) $T_{e \rightarrow z}$ is minimum.

- The closed-loop poles of the linearized plant are in a prescribed region $\mathbf{S}$ of the open left-half plane.

Our design objectives have the following LMI formulation.

\section{Convex optimization in LMI region}

The design problem of the application presented in this paper consists of computing a state-feedback gain $K$ that stabilizes the LPV system defined by (17) whose the varying parameter $\rho$ varies within a polytope that is defined thanks to Table I. Integral action is embedded in order to ensure no errors for the closed-loop tracking of angular references. The controller minimizes a $\mathrm{H}_{2}$ norm constraint for a set of vertices that define a convex polytope [10], [11].

Moreover, in order to take into account regional pole constraints, it is interesting to design the control $K$ such that the closed loop poles of $\left(A+B_{u} K\right)$ lie in a suitable subregion of the complex left-half plane. This region for control purposes, is the set $\mathbf{S}(\alpha, \epsilon, \beta)$ of the complex number $\sigma+j a$ such that:

$$
\sigma<-\alpha<0, \quad|\sigma+j a|<\epsilon \text { and } \tan (\beta) \sigma<-|a|
$$

Confining the closed loop poles in this region ensures a decay rate $\alpha$, a minimum damping ratio $\zeta=\cos (\beta)$ and a maximum undamped frequency $\omega_{d}=\epsilon \cdot \sin (\beta)$ ( $\beta$ in radian). The combination objectives of robust $\mathrm{H}_{2}$ control with regional pole constraints is characterized for $W=W^{T}$ and $Y=Y^{T}$ thanks to the following LMI formulation: If there exists symmetric $Y>0$, with $L=K Y$ such that:

$$
\min _{\{Y, L\}} \operatorname{trace}(W)
$$

such that for $i=1, \ldots, 5$ :

$$
\begin{gathered}
N_{i}(Y, L)+N_{i}^{T}(Y, L)+B_{e, i} B_{e, i}^{T} \leq 0 \\
\left(\begin{array}{ll}
Y & Y C_{z, i}^{T} \\
C_{z, i} Y & W
\end{array}\right) \geq 0 \\
N_{i}(Y, L)+N_{i}^{T}(Y, L)+2 \alpha Y<0
\end{gathered}
$$

$$
\left(\begin{array}{ll}
-\epsilon Y & N_{i}(Y, L) \\
N_{i}^{T}(Y, L) & -\epsilon Y
\end{array}\right)<0
$$

$\left(\begin{array}{ll}s(\beta)\left(N_{i}(Y, L)+N_{i}^{T}(Y, L)\right) & c(\beta)\left(N_{i}(Y, L)-N_{i}^{T}(Y, L)\right) \\ -c(\beta)\left(N_{i}(Y, L)-N_{i}^{T}(Y, L)\right) & s(\beta)\left(N_{i}(Y, L)+N_{i}^{T}(Y, L)\right)\end{array}\right)<0$

where $N_{i}(Y, L)=A_{i} Y+B_{u, i} L, s()=.\sin ($.$) and c()=$. $\cos ($.$) . In this study, the most important task is to find the$ variables $Y$ and $L$. Once a feasible solution $(Y, L)$ of $(20)$ is computed, the state-feedback gain matrix $K$ is synthesized as $K=L Y^{-1}$. Therefore, the control law is derived into statefeedback gains for the angular velocities and PI controllers for the angular tracking.

\section{SIMULATION RESULTS}

In this section, the performance and stability of the robust controller for angular velocity regulation and the flight reference tracking are presented by numerical simulations. In this sequel, the robustness of the proposed controller with respect the exogenous input $e(t)$ is not presented. The elements of the experimental benchmark which is based on a quadrotor Draganflyer are modeled and implemented. The further investigation in a practical sense will aim at presenting measurements and results in flight conditions.

\section{A. Flight tracking of angular positions for a set of parame- ters $\left(x_{C G}, m_{p}\right)$}

This paragraph presents nonlinear simulations. They are performed using the equations of section II. Actuator model and its dedicated control are also implemented.

For the simulations, we choose the parameter values $x_{C G}=$ 0.05 meter and the mass of the load $m_{p}=200$ grams. Those parameters are included in the convex polytope defined by the vertices in Table I. We specify the LMI region for the pole placements as the set $\mathbf{S}(\alpha, \epsilon, \beta)$ where the decay rate $\alpha=2$, $\beta=\frac{\pi}{4}$ for a minimum damping ratio $\zeta=\cos (\beta)=0.707$ and $\epsilon=40$.

Fig. 5 exhibits the tracking references for the pitch, roll and yaw angles. In Fig. 6, the results are presented. As can be observed, a settling time of about $2 \mathrm{~s}$ without overshoot is obtained for the angles. Fig. 7 and Fig. 8 illustrate the propeller speeds $\omega_{i}$ generated in the maneuver for the offcentered quadrotor. From the top view of Fig. 1, the front propeller speed $\omega_{1}$ is much higher than the one of the rear propeller $\omega_{3}$. By increasing the propeller speed $\omega_{1}$, the quadrotor succeeds in tracking the references despite the location of the payload $m_{p}$. Speeds of the propellers 2 and 4 are very similar as shown on Fig. 8.

\section{B. Discussion}

The proposed controller which is synthesized in LMI region meet the expected requirements of the control objectives. As Shown in Fig. 6, the controller ensures the tracking of references for a payload of mass $m_{p}=200$ grams where the center of gravity is such as $x_{C G}=0.05$ meter. The pole placement in LMI region proposes a fixed gain controller based on a state-feedback approach which considers time 


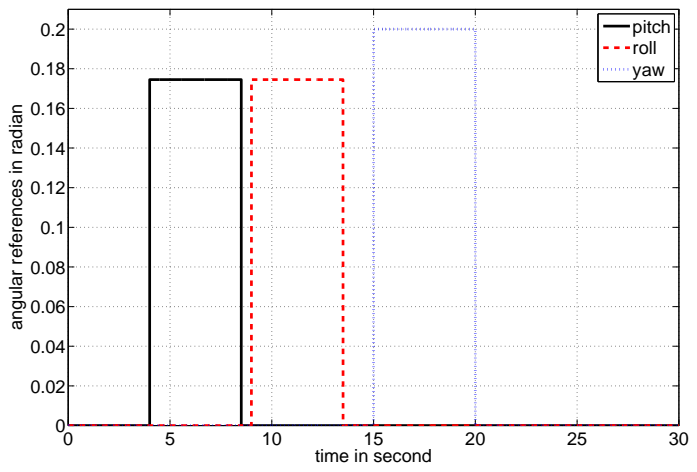

Fig. 5. Angular position references in pitch, roll and yaw

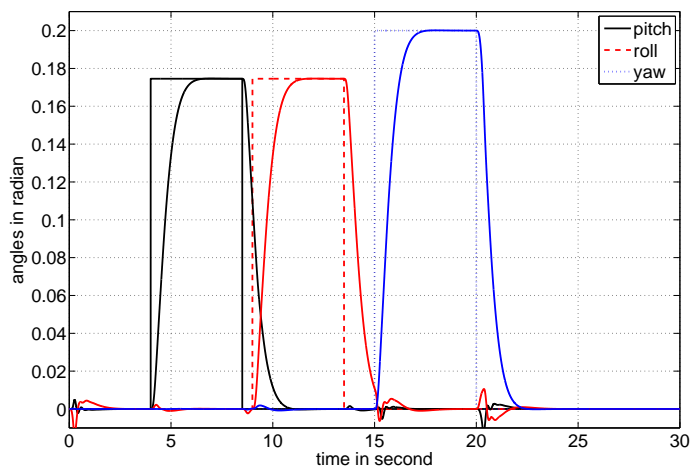

Fig. 6. Tracking of pitch, roll and yaw angles
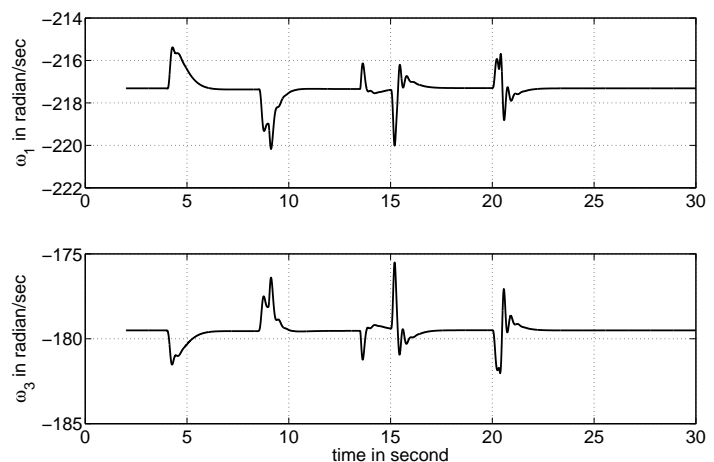

Fig. 7. Speeds of the propeller 1 (front) and 3 (rear)

response constraints thanks to the parameters $\alpha, \beta$ and $\epsilon$. The interesting point is that the obtained controller leads to similar performances for the closed loop plant in the polytope set of parameters $m_{p}$ and $x_{C G}$. One could expect better settling time for the angles taking into account the mechanical constraints of the propellers. Avoiding the saturations of the actuators, based on existing control strategies in the literature, the paper proposes an efficient solution which guarantees closed loop stability in LMI region and similar performances of the outputs in a specified set of known or uncertain parameters for an off-centered quadrotor.

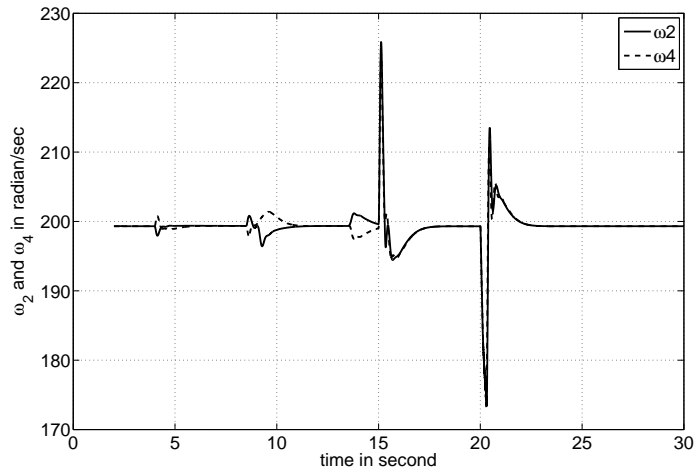

Fig. 8. Speeds of the propeller 2 (right) and 4 (left)

\section{REALIZATION}

A benchmark, based on the structure of a quadrotor Draganflyer has been designed. The core of the system is a dsPic40F13 microcontroller. This latter is programed with the Simulink Blockset Embedded Target microchip devices.This tool allows an easy and fast development [14], [15] from the Simulink environment without typing any C code lines. The system also includes:

- a control remote with a $41 \mathrm{MHz}$ four channels emitter and a $41 \mathrm{MHz}$ four channels receiver

- a miniature, gyro-enhanced Attitude and Heading Reference System XSENS MTI

- Four MOSFET PWM DC motor controllers

On the one hand, the reference attitude angles $\tilde{\phi}, \tilde{\theta}$ and the reference yaw rate $\tilde{r}$ are issued from the remote. To control the height, the pilot is in the loop. The transmitted PPM frame is decoded by the receiver and processed with the Input Capture module of the dsPic. On the other hand, the measured attitude angles and angular velocities are issued from the MTI with a RS232 format ( $25 \mathrm{~Hz}, 38400$ bauds). The presented control law is a state-feedback $K$ with PI controllers. The synthesis is described in section III-C. The control input signals are transformed into Pulse Width Modulation (PWM) thanks to the dsPic Output Compare in order to control the MOSFET $10 \mathrm{KHz}$ PWM DC motor controllers. Some flights have been completed with Proportionnal Derivative and Linear Quadratic Controllers. The proposed control law will be tested shortly and we expect to present experimental results during the oral presentation.

\section{CONCLUSION AND PERSPECTIVES}

Guided by the need to ensure trajectory tracking for a quadrotor which is dedicated to carry various payloads, the present paper proposes a mathematical description of the offcentered UAV and a simple way to control the outputs under time domain constraints.

The solution considers the variations of the mass $m_{p}$ of the load and the displacement along the $\mathrm{x}$-axle of the gravity center. The controller provides good performances for the set of parameters with respect stability and tracking. The results are proved to be efficient, compared to the ones usually 
implemented in such an UAV [3], [5], [8]. The paper does not bring the comparison of existing solutions.

Based on our own experimental UAV benchmark briefly presented in section $\mathrm{V}$, the perspective aims at testing the proposed control. In the same time, a scientific evolution investigates gain scheduling control for varying parameters during the mission of the UAV.

\section{REFERENCES}

[1] G. Hoffmann, H. Huang, S. Waslander and C Tomlin, "Quadrotor helicopter flight dynamics and control: Theory and experiment", in Proceedings of the AIAA Guidance, Navigation and Control Conference, Hilton Head, SC, 2007.

[2] P. Pounds, J. Gresham, R. Mahony, J. Robert and P. Corke, "Towards dynamically favourable quad-rotor aerial robots", in Proceedings of the Australian Conference on Robotics and Automation, Canberra, Australia, 2004.

[3] P. Pounds, R. Mahony and P. Corke, "Modelling and control of a quadrotor robot", in Proceedings of the Australian Conference on Robotics and Automation, Auckland, New-Zealand, 2006.

[4] D. Gurdan, J. Stumpf, M. Achtelik, K.-M Doth, G. Hirzinger and D. Rus, "Energy-efficient autonomous four-rotor flying robot controlled at 1khz", in IEEE Conference on Robotics and Automation, Roma, Italy, 2006, pp 361-366.

[5] D.B. Lee, T.C. Burg, B. Xian and D.M. Dawson, "Output feedback tracking control of and under-actuated quad-rotor UAV", in IEEE American Control Conference, New York, US, 2007.

[6] P. Castillo, R. Lozano and A. Dzul, Modelling and control of miniflying machines, Springer-Verlag, 2005.

[7] J-P. Bristeau, P. Martin, E. Salaum and N. Petit, "The role of propeller aerodynamics in quadrotor UAV", in 10th IEEE European Control Conference, Budapest, Hungary, 2009, pp 683-688.

[8] E. Altug, J. Ostrowski and R. Mahony, "Control af a quadrotor helicopter using visual feedback", in IEEE Conference on Robotics and Automation, Washington, US, 2009, vol. 1 pp 72-77.

[9] T. Hamel, R. Mahony, R. Lozano and J. Ostrowski, "Dynamic modelling and configuration stabilization for an X4-flyer", in Proceedings of the 15th IFAC World Congress, Barcelona, Spain, 2002.

[10] M. Chilali, P. Gahinet, $H_{\infty}$ design with pole placement constraints: an LMI approach, IEEE Transactions on Automatic Control, vol. 41 number 3, 1996, pp 358-367.

[11] C. Scherer, P. Gahinet, and M. Chilali, Multiobjective output-feedback control via LMI optimization, IEEE Transactions on Automatic Control, vol. 42 number 7, 1997, pp 896-911.

[12] J. Lofberg, "YALMIP: A toolbox for modeling and optimization in MATLAB", in IEEE CACSD Conference, Taipei, Taiwan, 2004, pp 284-289.

[13] J.F. Sturm, ”Using SeDuMi 1.02, a Matlab toolbox for optimization over symmetric cones", Optimization Methods and Software, vol 11, 1999, pp 625-653.

[14] S. Viollet, L. Kerhuel, and N. Franceschini, "A 1-gram dual sensorless speed governor for micro-air vehicles", in 16th Mediterranean Conference on Control and Automation, Ajaccio, France, 2008, pp 1270-1275.

[15] L. Kerhuel, "Embedded target for PIC/dsPIC", 2010.

\section{APPENDIX}

The center of gravity coordinates:

$$
\left(\begin{array}{lll}
x_{c g} & y_{c g} & z_{c g}
\end{array}\right)^{T}=\left(\begin{array}{lll}
\frac{m_{p} \alpha}{m} & \frac{m_{p} \beta}{m} & \frac{m_{p} \gamma}{m}
\end{array}\right)^{T}
$$

The transformation matrix $\mathbf{T}_{b E}$

$$
\left(\begin{array}{ccc}
\operatorname{co} \theta \operatorname{co} \psi & \operatorname{si\varphi } \operatorname{si\theta } \operatorname{co} \psi-\operatorname{co\varphi } \operatorname{si} \psi & \operatorname{co\varphi } \operatorname{si\theta } \operatorname{co} \psi+\operatorname{si\varphi } \operatorname{si} \psi \\
\operatorname{co\theta } \operatorname{si} \psi & \operatorname{si\varphi } \operatorname{si\theta } \operatorname{si} \psi+\operatorname{co\varphi } \operatorname{co} \psi & \operatorname{co\varphi } \operatorname{si\theta } \operatorname{si} \psi-\operatorname{si\varphi } \cos \psi \\
-\operatorname{si\theta } & \operatorname{si\varphi } \operatorname{co} \theta & \operatorname{co\varphi } \varphi \operatorname{co} \theta
\end{array}\right)
$$

For the motors, the cross and the payload which inertia moments are $\left\{I_{m_{x}}, I_{m_{y}}, I_{m_{z}}\right\},\left\{I_{c_{x}}, I_{c_{y}}, I_{c_{z}}\right\},\left\{I_{b_{x}}, I_{b_{y}}, I_{b_{z}}\right\}$ respectively. The inertia moments and products are calculated about the centre of gravity c.g. for the four motors $m_{1}, m_{2}$, $m_{3}, m_{4}$, the cross $I_{c}^{\prime}$ and the payload $I_{b}^{\prime}$.

$$
\begin{aligned}
& I_{m 1_{x}}=I_{m_{x}}+m_{m}\left(y_{c g}^{2}+z_{c g}^{2}\right) \\
& I_{m 1_{y}}=I_{m_{y}}+m_{m}\left(\left(l-x_{c g}\right)^{2}+z_{c g}^{2}\right) \\
& I_{m 1_{z}}=I_{m_{z}}+m_{m}\left(\left(l-x_{c g}\right)^{2}+y_{c g}^{2}\right) \\
& I_{m 1_{x y}}=m_{m}\left(l-x_{c g}\right) y_{c g} \\
& I_{m 1_{x z}}=m_{m}\left(l-x_{c g}\right) z_{c g} \\
& I_{m 1_{y z}}=m_{m} y_{c g} z_{c g} \\
& I_{m 2_{x}}=I_{m_{x}}+m_{m}\left(\left(l-y_{c g}\right)^{2}+z_{c g}^{2}\right) \\
& I_{m 2_{y}}=I_{m_{y}}+m_{m}\left(x_{c g}^{2}+z_{c g}^{2}\right) \\
& I_{m 2 z}=I_{m_{z}}+m_{m}\left(x_{c g}^{2}+\left(l-y_{c g}\right)^{2}\right) \\
& I_{m 2_{x y}}=m_{m} x_{c g}\left(l-y_{c g}\right) \\
& I_{m 2_{x z}}=m_{m} x_{c g} z_{c g} \\
& I_{m 2_{y z}}=m_{m}\left(l-y_{c g}\right) z_{c g} \\
& I_{m 3_{x}}=I_{m_{x}}+m_{m}\left(y_{c g}^{2}+z_{c g}^{2}\right) \\
& I_{m 3_{y}}=I_{m_{y}}+m_{m}\left(\left(x_{c g}+l\right)^{2}+z_{c g}^{2}\right) \\
& I_{m 3_{z}}=I_{m_{z}}+m_{m}\left(\left(x_{c g}+l\right)^{2}+y_{c g}^{2}\right) \\
& I_{m 3_{x y}}=m_{m}\left(x_{c g}+l\right) y_{c g} \\
& I_{m 3_{x z}}=m_{m}\left(x_{c g}+l\right) z_{c g} \\
& I_{m 3_{y z}}=m_{m} y_{c g} z_{c g} \\
& I_{m 4_{x}}=I_{m_{x}}+m_{m}\left(\left(l+y_{c g}\right)^{2}+z_{c g}^{2}\right) \\
& I_{m 3_{y}}=I_{m_{y}}+m_{m}\left(x_{c g}^{2}+z_{c g}^{2}\right) \\
& I_{m 4_{z}}=I_{m_{z}}+m_{m}\left(x_{c g}^{2}+\left(l+y_{c g}\right)^{2}\right) \\
& I_{m 4_{x y}}=m_{m} x_{c g}\left(l+y_{c g}\right) \\
& I_{m 4_{x z}}=m_{m} x_{c g} z_{c g} \\
& I_{m 4_{y z}}=m_{m}\left(l+y_{c g}\right) z_{c g} \\
& I_{c_{x}^{\prime}}=I_{c_{x}}+m_{c}\left(y_{c g}^{2}+z_{c g}^{2}\right) \\
& I_{c_{y}^{\prime}}=I_{c_{y}}+m_{c}\left(x_{c g}^{2}+z_{c g}^{2}\right) \\
& I_{c_{z}^{\prime}}=I_{c_{z}}+m_{c}\left(x_{c g}^{2}+y_{c g}^{2}\right) \\
& I_{c_{x y}^{\prime}}=m_{c} x_{c g} y_{c g} \\
& I_{c_{x z}^{\prime}}=m_{c} x_{c g} z_{c g} \\
& I_{c_{y z}^{\prime}}=m_{c} y_{c g} z_{c g} \\
& I_{b_{x}^{\prime}}=I_{b_{x}}+m_{b}\left(\left(y_{c g}-\beta\right)^{2}+\left(z_{c g}-\gamma\right)^{2}\right) \\
& I_{b_{y}^{\prime}}=I_{b_{y}}+m_{b}\left(\left(x_{c g}-\alpha\right)^{2}+\left(z_{c g}-\gamma\right)^{2}\right) \\
& I_{b_{z}^{\prime}}=I_{b_{z}}+m_{b}\left(\left(x_{c g}-\alpha\right)^{2}+\left(\left(y_{c g}-\beta\right)^{2}\right)\right. \\
& I_{b_{x y}^{\prime}}=m_{b}\left(x_{c g}-\alpha\right)\left(y_{c g}-\beta\right) \\
& I_{b_{x z}^{\prime}}=m_{b}\left(x_{c g}-\alpha\right)\left(z_{c g}-\gamma\right) \\
& I_{b_{y z}^{\prime}}=m_{b}\left(y_{c g}-\beta\right)\left(z_{c g}-\gamma\right)
\end{aligned}
$$

The inertia matrix coefficients about $c . g$. are:

$$
\begin{aligned}
J_{x, y, z} & =\sum_{i=1}^{4} I_{m_{i x, y, z}}+I_{c_{x, y, z}^{\prime}}+I_{b_{x, y, z}^{\prime}} \\
J_{x y, x z, y z} & =\sum_{i=1}^{4} I_{m_{i x y, x z, y z}}+I_{c_{x y, x z, y z}^{\prime}}+I_{b_{x y, x z, y z}^{\prime}}
\end{aligned}
$$

